\title{
Event driven 4D STEM acquisition with a Timepix3 detector: microsecond dwell time and faster scans for high precision and low dose applications
}

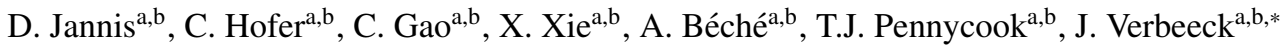 \\ ${ }^{a}$ EMAT, University of Antwerp, Groenenborgerlaan 171, 2020 Antwerp, Belgium \\ ${ }^{b}$ NANOlab Center of Excellence, University of Antwerp, Groenenborgerlaan 171, 2020 Antwerp, Belgium
}

\begin{abstract}
Four dimensional scanning transmission electron microscopy (4D STEM) records the scattering of electrons in a material in great detail. The benefits offered by 4D STEM are substantial, with the wealth of data it provides facilitating for instance high precision, high electron dose efficiency phase imaging via center of mass or ptychography based analysis. However the requirement for a 2D image of the scattering to be recorded at each probe position has long placed a severe bottleneck on the speed at which 4D STEM can be performed. Recent advances in camera technology have greatly reduced this bottleneck, with the detection efficiency of direct electron detectors being especially well suited to the technique. However even the fastest frame driven pixelated detectors still significantly limit the scan speed which can be used in 4D STEM, making the resulting data susceptible to drift and hampering its use for low dose beam sensitive applications. Here we report the development of the use of an event driven Timepix 3 direct electron camera that allows us to overcome this bottleneck and achieve 4D STEM dwell times down to $100 \mathrm{~ns}$; orders of magnitude faster than what has been possible with frame based readout. We characterise the detector for different acceleration voltages and show that the method is especially well suited for low dose imaging and promises rich datasets without compromising dwell time when compared to conventional STEM imaging.
\end{abstract}

Keywords: Scanning transmission electron microscopy, 4D STEM, Low dose, Event based detection

\section{Introduction}

The development of direct pixelated detectors has revolutionized the imaging capabilities of multiple scientific fields, from particle tracking to X-ray and electron microscopy [1, 2]. In electron microscopy (EM), the efficiency of direct detectors has provided a leap forward in the dose efficiency possible in transmission electron microscopy (TEM). For fields such as molecular biology the resolution provided by EM is limited more by the poor signal achievable using the extremely low doses required to avoid unacceptable damage to the material than by the electron optics themselves. For such fields therefore dose efficiency is of the upmost importance, and the greatly enhanced dose efficiency provided by direct electron detectors made possible the so called resolution revolution in cryo-EM [3, 4].

The most fragile dose sensitive samples, such as proteins, have largely been the preserve of conventional

\footnotetext{
*Corresponding author

Email address: jo.verbeeck@uantwerp. be (J. Verbeeck)
}

phase contrast TEM imaging as in cyro-EM. Phase contrast imaging in STEM has historically been far less dose efficient, but advanced STEM methods that also provide highly dose efficient phase contrast signals have been developed and also benefited greatly from advances in pixelated detector technology [5]. The vastly expanded information on the position dependence of the electron scattering provided in 4D STEM by capturing a $2 \mathrm{D}$ image of the scattering at each probe position provides advantages that go well beyond the much greater flexibility it provides in choosing between conventional STEM imaging modalities after taking the data [6, 7]. The richness of 4D STEM datasets also allow for significantly more advanced analysis. For example, center of mass (COM) and ptychographic based 4D STEM methods both provide greatly enhanced sensitivity to the local potentials of a material and greatly enhanced signal per electron compared to conventional STEM methods [8, 6, 9]. Moreover ptychographic methods have been shown capable of also providing significantly enhanced dose efficiency over conventional TEM phase contrast [10], thus offering the prospect of 

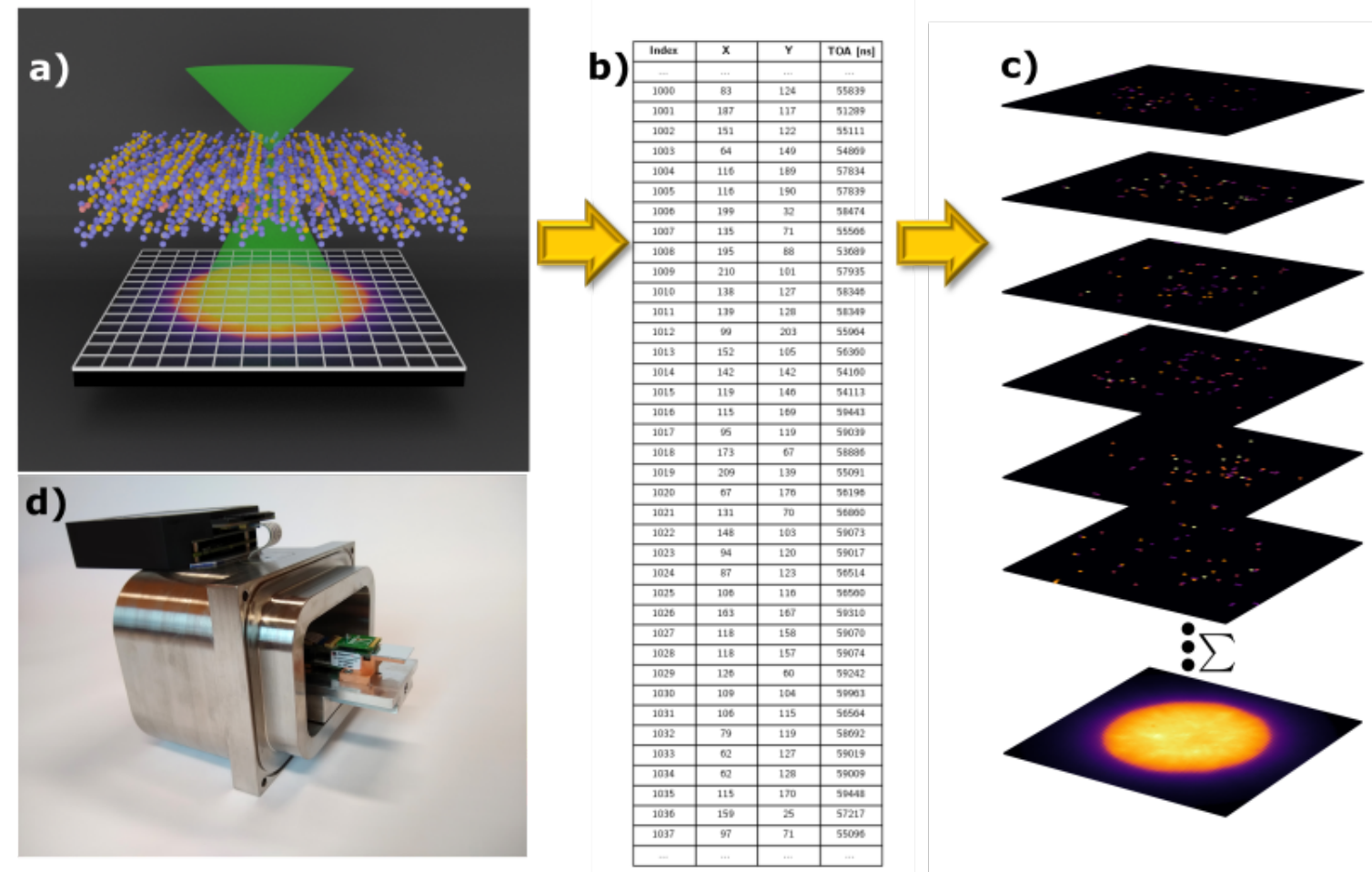

Figure 1: (a) The schematic setup, where a convergent electron beam is scanned across the sample and during the scan, the point of impact and TOA is measured for each incoming electron. (b) The data list created by the detector where for every event the point of impact and TOA is indicated. (c) When the probe position at each time is accurately known, a diffraction pattern belonging to the time range for a given probe position can be obtained resulting in a full 4D STEM dataset. (d) Custom built retractable assembly to place the Timepix 3 detector in the electron microscope column.

another leap forward in low dose performance as well as a greater precision, for example for mapping local charge densities [11, 12, 13].

4D STEM methods such as COM or ptychography do not require cameras with particularly large numbers of pixels [9, 14]. Given the achievable frame rate depends on the number and bit depth of the pixels due to finite readout and data transfer rates, the development of small direct electron detectors has greatly benefited 4D STEM. Small fast hybrid pixel direct electron detectors such as the Medipix3 [15] are particularly attractive as they also offer the benefit of beam hardness, with the detection layer physically separated from the readout electronics. However, such detectors have typically been employed at frame rates of at most a few thousand frames per second [16].

By utilizing the much reduced bandwidth required for a 1-bit counting depth in each pixel of a Medipix3 detector, O'Leary et al. demonstrated a significant speedup of 4D STEM acquisition at a $12.5 \mathrm{kHz}$ frame rate, corresponding to an 80 microsecond dwell time [17, 18].
Although this allowed them to achieve a relatively low dose of $200 \mathrm{e}^{-} \AA^{-2}$ by using an extremely low probe current in a focused probe configuration, such a dose is still much higher than required by the most beam sensitive materials [19, 20, 21] and the speed is still far slower than the single to few microsecond dwell times used in rapid conventional ADF based STEM measurements. Detector technology continues to advance and achievable frame rates are increasing. For example 11 microsecond dwell time 4D STEM has recently been achieved with a specially designed custom extremely high frame rate camera [22, 23], but this is still an order of magnitude slower than the single to very few $\mu$ s typical for rapid scanning STEM, as frequently used in drift corrected time series of STEM scans..

By using a defocused probe the CBED pattern contains information from a larger area of the sample from each probe position. Although significant overlap of the illuminated sample areas from adjacent probe positions is generally required, the reduced real space sampling requirements allows defocused probe ptychogra- 
phy techniques [24, 25] to more easily reach low doses with relatively slow cameras compared to focused probe techniques. This advantage has recently been utilized to demonstrate defocused probe electron ptychography at extremely low cryo-EM doses with a Medipix3 [26], showing advantages over conventional TEM common to ptychographic techniques such as the single signed contrast transfer function (CTF) with a passband optimizable via the convergence angle and without the need for additional aberrations. However defocused probe techniques preclude the simultaneous use of the highly informative ADF based Z-contrast signal and generally involve solving for the phase using iterative convergence with the accompanying risks of incorrect convergence particularly at low doses. The focused probe single side band (SSB) [27, 6] and the Wigner distribution deconvolution (WDD) [28, 29] methods can provide simultaneous Z-contrast signals and non-iterative phase determination, and with progressing camera speedup can also now reach the extreme low dose regime of cryo-EM.

Here we demonstrate the use of an alternative event driven detector architecture to completely remove the bottleneck in speed for 4D STEM in comparison to conventional STEM detectors based on scintillators and photomultipliers for the low probe currents desirable for low dose operation. The event driven Timepix 3 detector was developed with an emphasis on time resolution for applications in a wide range of scientific disciplines with a maximum time resolution of $1.56 \mathrm{~ns}$ [30, 31]. Event driven operation allows one to take advantage of event sparsity to achieve enhanced time resolution by avoiding the readout of pixels containing zero counts. In STEM one can reduce the dose imposed on a sample per unit area by reducing the probe current and dwell time, both of which increase the sparsity of the events in each probe position. We present results from 4D STEM performed with a Timepix3 detector at dwell times of a few microseconds down to $100 \mathrm{~ns}$ at both 60 and $200 \mathrm{kV}$ accelerating voltages. Although the detector configuration used herein requires the use of low probe currents, we show how the speed facilitates multiply scanned 4D STEM to be used in order to increase the signal-to-noise with minimal susceptibility to drift. The results demonstrate that event driven camera technology enables the efficiency of 4D STEM and in particular electron ptychography to be exploited for both large fields of view and the extremely low doses without resorting to a defocused probe configuration.

\section{Experimental Setup}

The event based detector used in the camera setup in this work is the AdvaPIX TPX3 [32] which is a Timepix 3 based detector where the thickness of the sensitive silicon layer is $300 \mu \mathrm{m}$. Timepix 3 chips are 3 -side tileable with a maximum event capacity of $0.43 \times 10^{6} / \mathrm{mm}^{2} / \mathrm{s}$. A single Timepix 3 chip contains $256 \times 256$ pixels where each pixel has a size of $55 \times 55$ $\mu \mathrm{m}$, resulting in a theoretical capacity of $80 \times 10^{6}$ counts per second for a single chip device. However due to the bandwidth of the USB 3.0 port used for the readout from our device to the computer, the maximum capacity for our single chip setup is $40 \times 10^{6}$ counts per second when using flat field illumination. If one electron lights up one pixel, this corresponds to a current of $6.4 \mathrm{pA}$ which compared to conventional STEM imaging is somewhat on the low side. In the Section 3 it is shown that this estimate has to be adjusted with more technical details but the order of magnitude is correct [33]. In Fig.11(a), a schematic view of the setup is shown where an incoming convergent electron beam interacts with the sample. After the interaction with the sample, the electron beam propagates into the far field regime where the detector is placed. In Fig. 11(b) an example of the data is shown where for every incoming event its point of impact and time of arrival (TOA) are saved. Knowing the probe position at each time, the conventional 4D STEM dataset can be obtained. In Fig. 1 (c) some measured diffraction patterns are shown in which the sparsity is seen due to the low dose per scanned point. The resulting position averaged convergent beam electron diffraction (PACBED), calculated from the sum of all diffraction patterns, is also shown.

The Timepix 3 detector was mounted on a probecorrected FEI Themis $\mathrm{Z}$ using a custom designed retractable mount interface shown in Fig. 11(d). In order to synchronize the scan coils with the detector, a custom scan engine is used [34, 35]. The detector and scan engine are synchronized by using a $10 \mathrm{MHz}$ reference clock output which is then multiplied with a custom designed phase locked loop circuit to act as a 40 $\mathrm{MHz}$ master clock for the Advapix in order to keep both scan engine and camera synchronised in time. At the start of the acquisition, a synchronisation signal is generated by the scan engine which triggers a recording sequence on the detector side. In this work, no flyback time is applied since we aim to reduce the dose as much as possible and we do not have access to a fast enough beam blanker to shut the beam down. Both the detector and scan engine have an application programming interface (API) in Python3 giving us the ability to auto- 
mate the acquisition sequence. The incoming raw data from the detector is processed using self written software and the ptychographic reconstruction is performed using the single side band ptychography reconstruction algorithm. The full recorded dataset and data processing software is made available in Zenodo allowing others to duplicate our efforts or to experiment with new algorithms that are optimised for event based data streams [36].

\section{Timepix3 Characterization}

The Timepix 3 detector is a hybrid direct electron detector where the silicon sensor layer is bonded onto the underlying processing ASIC electronics similar to the Medipix chips [37, 38]. As for the Medipix detectors, the main parameter to be changed is the energy threshold which is a pre-set discrimination level used to discriminate an electron event above the noise floor. Hence, by varying this parameter we can select the amount of signal detected for a particular beam current. The energy threshold influences mainly two things, firstly the amount of detected events when one electron hits the detector and secondly the number of detected electrons. Throughout the rest of the paper the amount of pixels which are excited by a single electron will be referred to as a cluster. The identification of the clusters is derived from the work by van Schayck et al. [39] where clusters are identified by checking if neighbouring pixels are excited within a small time interval ( $\sim 100 \mathrm{~ns})$. The energy threshold also influences the number of detected electrons because increasing the threshold will results in some events remaining undetected since they do not exceed the threshold value when scattering inside the sensitive silicon layer.

The camera used in this work shows a workable calibrated threshold range between 7 and $33 \mathrm{keV}$, in Supplementary Material S1 flat field images of different threshold values are shown where the artefacts for lower and higher threshold values are indicated. Note that these settings depend on an internal calibration routine that is performed when producing the detector [40, 41, 42]. During the experiments it was observed that the temperature of the detector chip remained constant around $30^{\circ}$.

In Fig. 2(a), the cluster size as a function of energy threshold is plotted for 60 and $200 \mathrm{kV}$ acceleration voltages. As expected, the cluster size decreases as a function of increasing energy threshold where for $200 \mathrm{kV}$ the lowest cluster size is approximately 2.2 pixels and for $60 \mathrm{kV}$ the size drops to 1 pixel at a threshold of $33 \mathrm{keV}$. In Fig. 2 (b), the detected electron current is

\begin{tabular}{|c|c|c|}
\hline & $\begin{array}{c}\text { Extrapolated fluorescent } \\
\text { screen current }\end{array}$ & $\begin{array}{c}\text { Timepix3 } \\
\text { current }(7 \mathrm{keV})\end{array}$ \\
\hline $60 \mathrm{kV}$ & $0.1 \pm 0.01 \mathrm{pA}$ & $0.1 \mathrm{pA}$ \\
\hline $200 \mathrm{kV}$ & $0.1 \pm 0.01 \mathrm{pA}$ & $0.09 \mathrm{pA}$ \\
\hline
\end{tabular}

Table 1: The measured extrapolated current on the fluorescent screen and on the Timepix 3 operated at a threshold of $7 \mathrm{keV}$. This small current was measured on the fluorescent screen by first measuring the current of a large parallel beam. Afterwards, a small aperture is inserted to block a large part of the beam. The current through the aperture can be calculated from knowing the radii of both the parallel beam and aperture. The error on the extrapolated fluorescent screen is approximated to be $10 \%$.

shown as a function of energy threshold for the two acceleration voltages. The detected electron current is calculated by dividing the number of events on the detector by the cluster size within a fixed amount of time (two seconds in this experiment). From Fig.2(b), it is seen that at higher energy thresholds $(33 \mathrm{keV})$ for $60 \mathrm{kV}$, a significant amount of electrons ( $25 \%$ at $33 \mathrm{keV})$ remain undetected.

Since the incoming electron current is lower than the detection threshold for measuring the current via the fluorescent screen or spectrum drift tube method, it was not possible to measure the current during the characterization experiments. Therefore, an additional measurement was performed to verify that the detector is not operated in an undercounting regime. This was done by using a parallel beam with sufficient current (30 pA) such that the fluorescent screen is able to measure it. Next a small aperture was inserted from which the remaining current can be estimated via the radii of both the original beam and the apertured beam assuming a homogeneous current density in the original beam. The apertured beam is then placed on the detector from which the number of electrons detected can be measured. A threshold of $7 \mathrm{keV}$ is used to be sure that the least amount of electrons are lost. The result is shown in Table 1 where it is observed that the measured extrapolated current from the fluorescent screen and the Timepix 3 are very similar indicating that the Timepix 3 was not operated in undercounting mode. The error on the current measurement from the fluorescent screen is approximated to be around 10\% [33]. A faraday cup could be used to get a more precise measurement on the actual current but was unavailable for our experiments. Moreover in the work of Krause et al. [33] it is also shown that direct electron detectors provide an accurate estimate for low currents in range of acceleration voltages used during the experiments.

Since the $7 \mathrm{keV}$ threshold loses the least amount of electrons (see Fig. 2), this threshold is the most accu- 

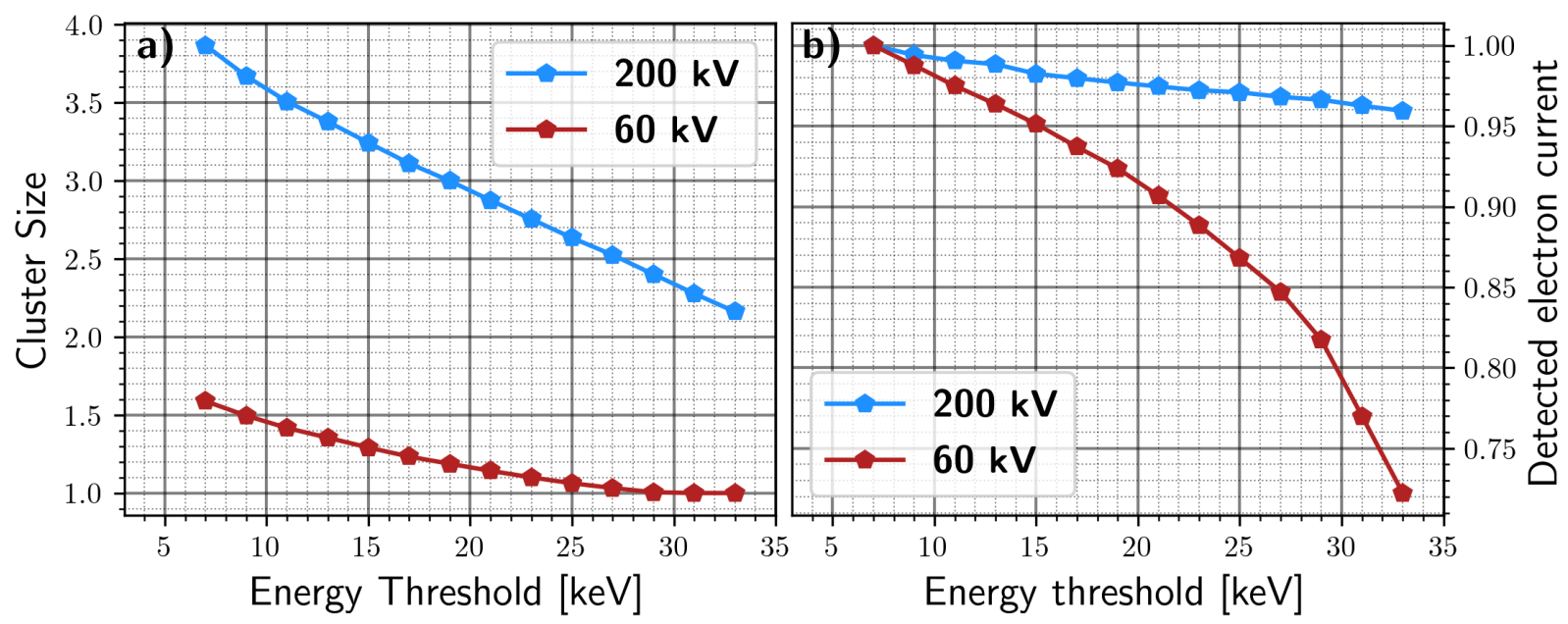

Figure 2: (a) The cluster size as a function of energy threshold for the two acceleration voltages 60 and $200 \mathrm{kV}$. (b) The detected electron current which is calculated by dividing the number of events per second on the detector by the average cluster size. The detected current is relative compared to the detected current at a energy threshold of $7 \mathrm{keV}$.

rate measurement of the incoming electron count rate. Note that this is a lower boundary on the actual current since some electrons will backscatter and other electrons will scatter inside the silicon layer without exceeding the threshold energy. Next to the backscattering of the incoming electrons, electron counts can be lost due to the dead time of the individual pixels which is $\sim 500 \mathrm{~ns}$. For the flat field illumination acquisition, the detected incoming electron count rate is $14 \times 10^{6}$ counts/s $(2.4 \mathrm{pA})$ and $6 \times 10^{6}$ counts/s $(1 \mathrm{pA})$ for respectively 60 and $200 \mathrm{kV}$ at a threshold of $7 \mathrm{keV}$. Therefore the probability for an electron to arrive in the same pixel area within $500 \mathrm{~ns}$ is in the order of $0.01 \%$ which is a very small fraction. When the illumination area decreases or the incoming current increases, the electrons missed due to the finite dead time of the individual pixels will increase and can become measurable [43]. In the rest of this work, the total incoming electrons are calculated by dividing the number of detected events by the cluster size which is then multiplied by the ratio between the electron current at $7 \mathrm{keV}$ and the used threshold value (see Fig. 2).

For example if $1 \times 10^{6}$ events per second are detected with a threshold of $30 \mathrm{keV}$ at a $60 \mathrm{keV}$ accelerating voltage, the incoming electron current is calculated by multiplying this value by $1 / 1.02$, the inverse of the cluster size, and 0.79 , the ratio between the detector electron current at 7 and $30 \mathrm{keV}$.

From Fig. 2 we can conclude that at this lowest threshold, more electrons are detected but they make on average a larger cluster size reducing the maximum cur- rent for which reliable detection and readout can be achieved. At $200 \mathrm{kV}$, the amount of events at the highest threshold ( $33 \mathrm{keV})$ is only $4 \%$ less compared than when using a threshold of $7 \mathrm{keV}$ while it significantly reduces the cluster size. This results in a higher allowable beam current as compared to lower threshold levels since there is an upper limit on the maximum count rate of the detector.

The detector was also briefly tested with an acceleration voltage of $300 \mathrm{kV}$. The cluster size increased quite considerably to an average of six pixels at a threshold of $33 \mathrm{keV}$. This meant that the incoming current should be dropped by a factor of three compared to $200 \mathrm{kV}$ which makes the use of $300 \mathrm{keV}$ electrons much less attractive and is therefore not considered further.

When increasing the threshold it is expected that the detected counts do not arise exactly at the position where the electron enters since the electron loses most of its energy towards the end of its track. Hence even when the average cluster size is one, it is expected that the modulation transfer function (MTF) will not approximate the theoretical limit of a square pixel [44]. The identification of clusters can be used to increase the MTF of the detector as shown in the work of van Schayck et al. [39] where they trained a neural network to better estimate the point of initial impact. However for our work such increasing of the MTF will not improve the results from our 4D STEM measurements since the algorithms used to reconstruct the signal are not sensitive to this [9, 14] . In Supplementary Material $S 2$, the corrected electron signal, where the point of im- 
pact is calculated using mean $\mathrm{x}$ and $\mathrm{y}$ coordinate of the cluster, is used to investigate if the integrated center of Mass (iCOM) result changes due to this improvement of MTF. In Supplementary Material S2 it is shown that a small discrepancy of approximately $1 \%$ between both reconstructed signals is observed. Therefore, when determining the positions of atoms and defects is the goal, declustering is not necessary, but when the aim is to obtain a quantitative iCOM signal then declustering is likely desirable. During the experiments, it was noted that the detector does not record the incoming signal for short periods of time ranging from $50 \mu \mathrm{s}$ to $1.75 \mathrm{~ms}$. This happens for $\sim 0.12 \%$ of the time that the detector is recording. This artefact is shown in Supplementary Materials S3. Up until now the origin of this artefact is not known but it is not problematic since it only occurs for such a small amount of time. In this work we took the simple approach of replacing the integrated signal of a particular probe position, where the detector is not recording, by the average signal. In the future better ways of reconstructing these signals can be performed, and hopefully the losses fully avoided in the first place, but this is outside the scope of this work. Since the amount of lost electrons is relatively small, this artifact does not significantly hinder the performance of the camera.

\section{Results \& Discussion}

A $2 \mathrm{D} \mathrm{WS}_{2}$ sample was used to showcase the performance of the detector at $60 \mathrm{kV}$. A multi-frame acquisition is performed where the probe, with a convergence angle of $25 \mathrm{mrad}$, is scanned over $1024 \times 1024$ probe positions three times at a dwell time of $6 \mu \mathrm{s}$. The dose per frame is estimated to be $6000 \mathrm{e}^{-} \AA^{-2}$. The size of the dataset is $6.6 \mathrm{~GB}$ which is a significantly lower data storage requirement than using a 1-bit mode of an equivalently sized frame-based detector where the data size would be 33 GB.

In recent years, another type of data storage for frame based detector has been developed. The compression method is named electron-event representation (EER) which stores each electron-detection event as a tuple of position and time [45]. This is similar to the output of event based detectors. However the speed of the framebased camera is still determined by the fps which is not the limit for the event based detectors [46, 47].

During the acquisition, the signal from the HAADF detector was read out simultaneously to collect the electrons which scatter to higher angles than the pixelated detector and provide a simultaneous Z-contrast image.
In this work we provide three different signal reconstruction methods which are annular bright field (ABF), where only events which land on a annular ring on the detector is integrated from 33 to $100 \%$ relative to the convergence angle in order to obtain the image. Secondly, the iCOM signal is reconstructed from calculating the centre-of-mass of each probe position on which a two dimensional integration is performed which yields a scalar image. Finally, the single side-band (SSB) method is used to retrieve the phase of the object. The $\mathrm{ABF}$ and $\mathrm{iCOM}$ signals are calculated directly from the event list (see Fig. 11(b)) whereas for the SSB reconstruction the data was converted to standard non-sparse fourdimensional data for the most facile input to our existing ptychographic processing software, which was written with framing cameras in mind. In the future the algorithms can be modified to accept the sparse event data directly or even perform 'live' imaging [47]. The resulting signals (ABF, iCOM and SSB) created from the 4D STEM images are shown in Fig. 3. The HAADF signal in Fig. 3(a,e) arises from the conventional HAADF detector (47-210 mrad) which we recorded in parallel with the event based detector data. The reconstructed multi-frame images are aligned using an open-source software which was developed for low signal-to-noise cryo-STEM data [48]. The software uses all possible combinations of image correlations, instead of using a single reference image, to determine the optimal shift. The relative image distortions between the scans are calculated using the SSB images due to its good contrast. The resulting images shifts were then also applied to the HAADF, ABF and iCOM images to align them. In Fig. $3(b, f)$ the ABF signal is shown where the low contrast arises as a result from the low dose conditions. This is not particularly surprising as in the weak phase object approximation (WPOA) zero contrast is expected when using a centrosymmetric detector configuration, and the $2 \mathrm{D} \mathrm{WS}_{2}$ is of course thin, relatively weak and lacking in channeling contrast in comparison to typical 3D materials [6].

The iCOM signal shown in Fig. 3 (c,g) and the SSB ptychographic reconstruction shown in Fig. 3 $(\mathrm{d}, \mathrm{g})$ both take greater advantage of the 4D data and show much stronger signals. The difference in contrast of the SSB with respect to the iCOM signal arises from the different contrast transfer functions (CTF) of both signals [8, 49] and the way in which the ptychographic method keeps track explicitly of where in probe reciprocal space each frequency is transferred [6, 9]. The lower limit of the electron dose is calculated using the information from Fig. 2 (b) where the experiment was performed at a energy threshold of $30 \mathrm{keV}$ indicating that minimally 


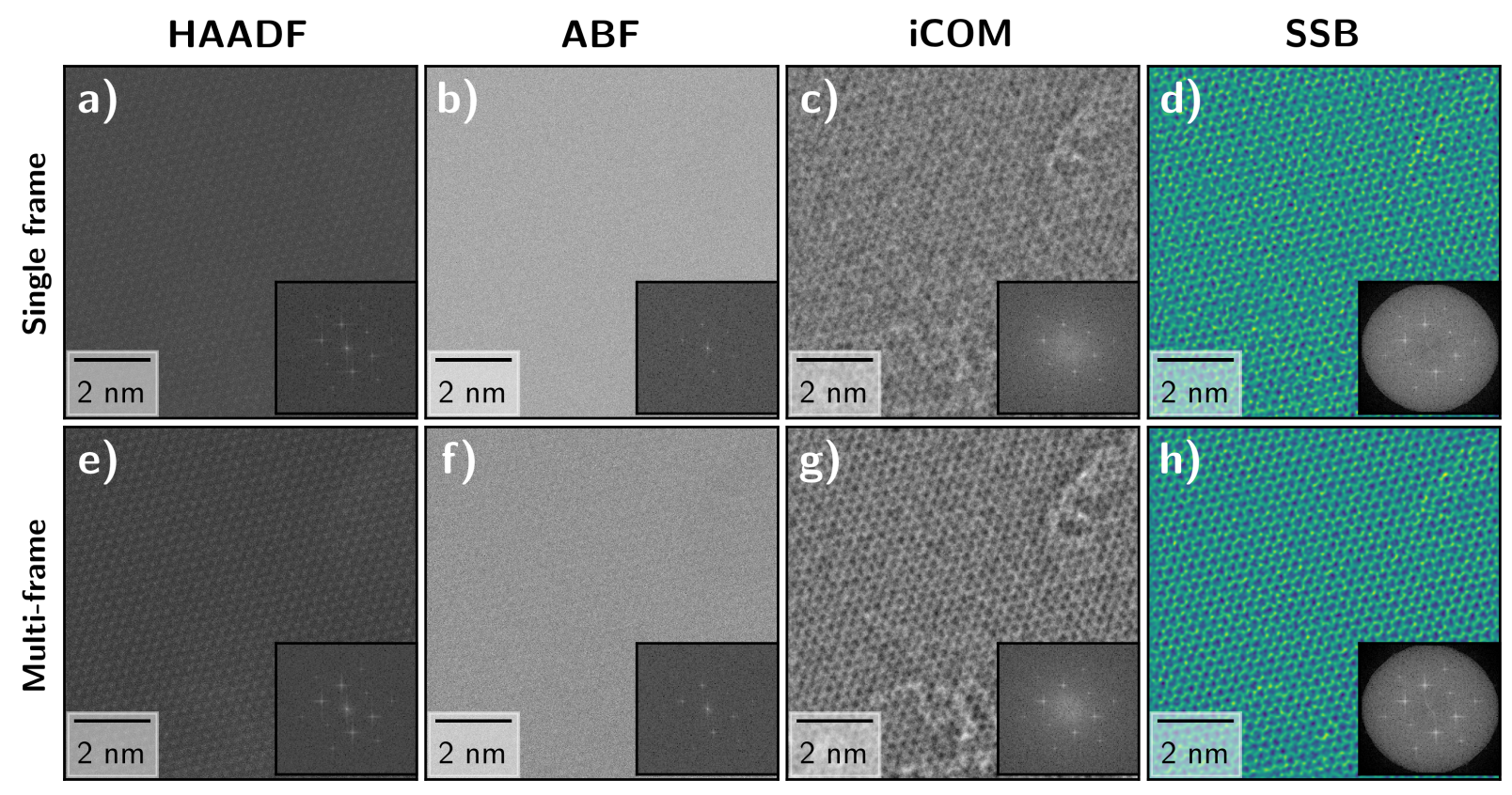

Figure 3: (a,e) Single and summed multi-frame HAADF images from a $3 \times 1024 \times 1024$ scan at $6 \mu$ s dwell time. The acceleration voltage used during the acquisition is $200 \mathrm{kV}$. The sample is a monolayer of $\mathrm{WS}_{2}$. The HAADF signal is collected with the conventional HAADF detector. (b,f) Single and multi-frame ABF images reconstructed from the simultaneously acquired 4D STEM dataset. (c,g) The reconstructed iCOM images from the single and multi-frame scans. (d,h) The reconstructed SSB images from the single and multi-frame scans.

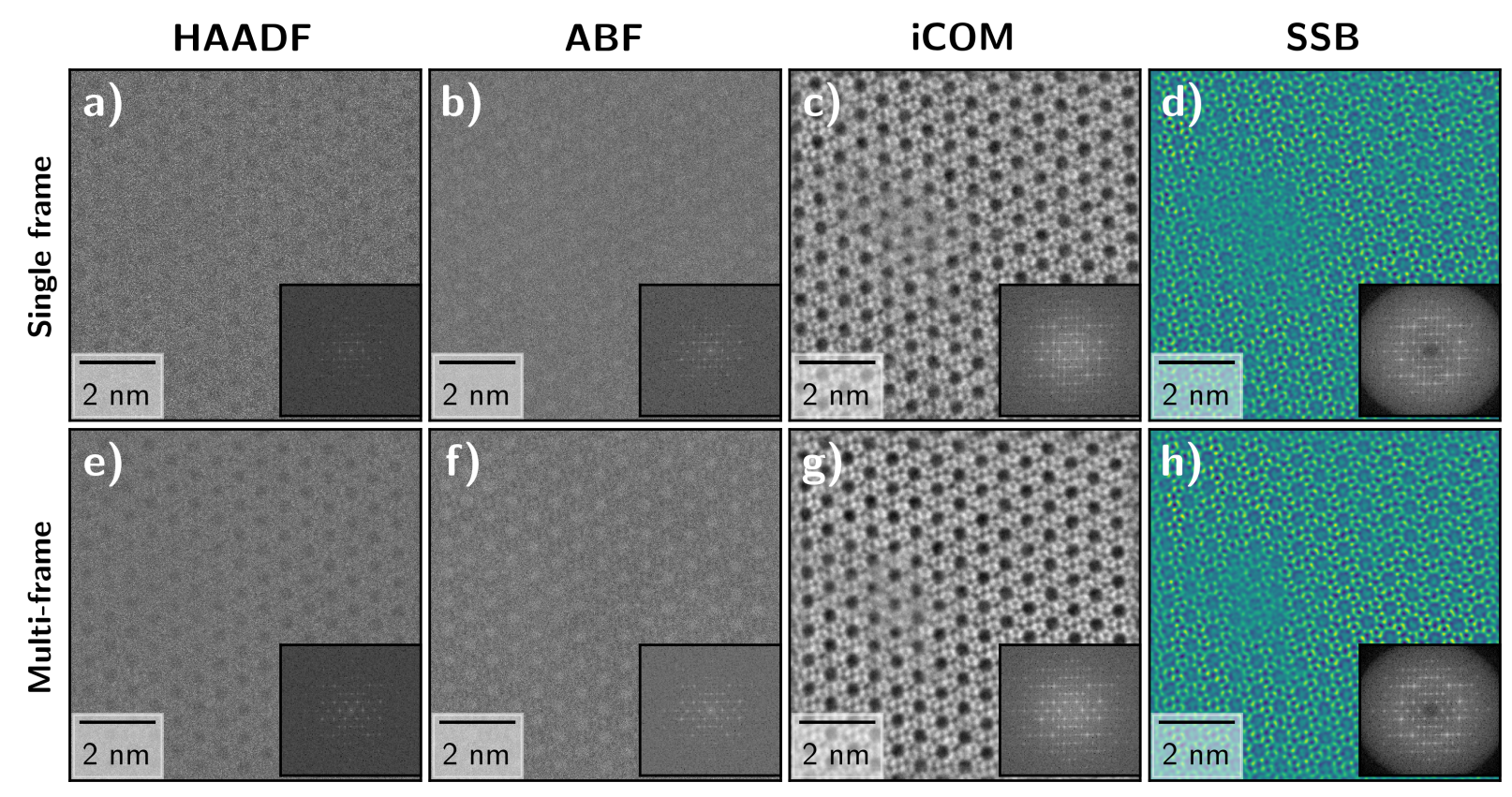

Figure 4: (a,e) Single and summed multi-frame HAADF images from a $1 \mu$ s dwell time $10 \times 1024 \times 1024$ scan of a silicalite- 1 zeolite sample. The acceleration voltage used during the acquisition is $60 \mathrm{kV}$.(b,f) Single and summed multi-frame ABF images reconstructed from the use of virtual detector. $(\mathbf{c}, \mathbf{g})$ The reconstructed iCOM images from the single and multi-frame scans. On the left of the image the distortions due to finite response time of the scan coils are visible. (d,h) The reconstructed SSB images from the single and multi-frame scans. 

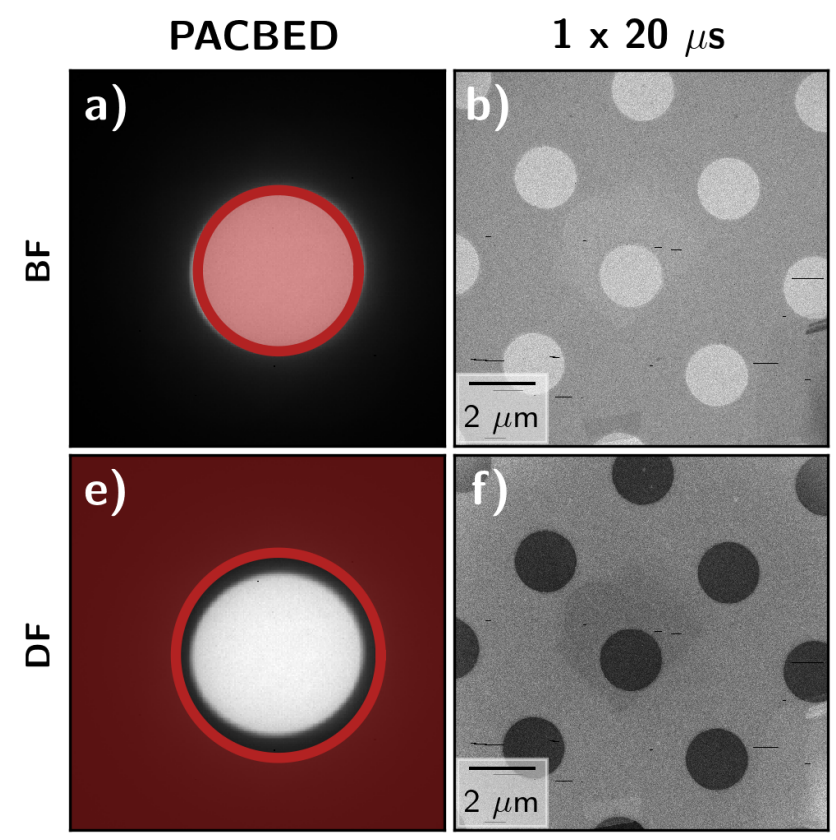

$200 \times 100 \mathrm{~ns}$
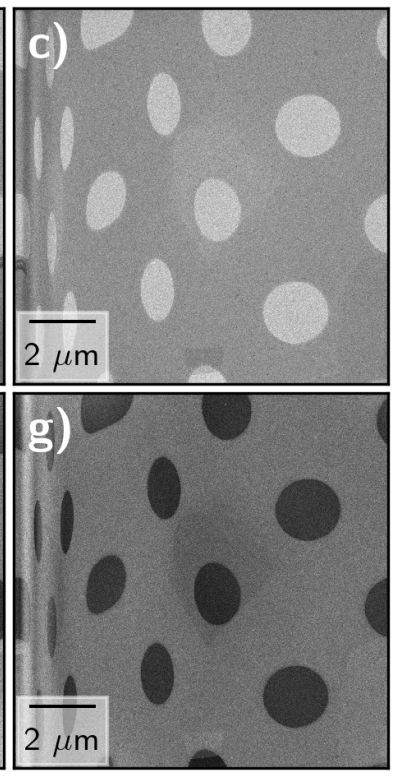

$1 \times 100 \mathrm{~ns}$

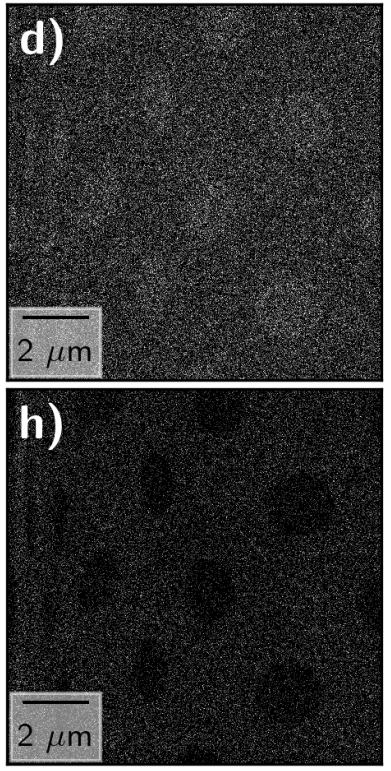

Figure 5: (a,e) The PACBED of the 4D STEM scan where, for both signals bright field (BF) and dark field (DF), the virtual detectors are shown. (b,f) The BF and DF signal of a $1024 \times 1024$ scan at $20 \mu$ s dwell time where the sample is a low magnification image a holey carbon film. The acceleration voltage used during the acquisition is $60 \mathrm{kV}$. (c,g) The summed BF and DF image of the same scan size with a dwell time of $100 \mathrm{~ns}$ and 200 frames which are scanned. Clear distortions arising from the response time of the coils are visible but the detector has no significant issues with this dwell time. (d,h) A single BF and DF frame of the fast scan using a dwell time of $100 \mathrm{~ns}$.

$\sim 23 \%$ of the incoming electrons are not detected. This gives a minimum dose of $\sim 6000 \mathrm{e}^{-} \AA^{-2}$ per frame where the incoming electron current used was $4.4 \mathrm{pA}$. The real dose used during the acquisition would be slightly higher since there is a small fraction of the electrons which is not detected due to the backscattering and pixel dead time. However as derived from Table 1] it is expected that the deviation between detected events and actual beam current is $\pm 10 \%$.

The same type of data acquisition at $60 \mathrm{kV}$ can be performed at $200 \mathrm{kV}$ where the main difference is the larger cluster size at $200 \mathrm{kV}$. However in terms of lost electrons, the $200 \mathrm{kV}$ is better since only a small fraction (3\%) seems to be lost by increasing the energy threshold to the $31 \mathrm{keV}$ used for the $200 \mathrm{kV}$ data presented here taken of a silicalite- 1 zeolite [50]. A $10 \times 1024 \times 1024$ probe position scan is performed at a dwell time of $1 \mu \mathrm{s}$ where the dose per frame was $300 \mathrm{e}^{-} \AA^{-2}$ and the total dose over the ten scans was $3000 \mathrm{e}^{-} \AA^{-2}$. The current used during the acquisition was $1.2 \mathrm{pA}$. The convergence angle used during the experiment was $12 \mathrm{mrad}$. In Fig. 4, the reconstructed signals are shown where the HAADF and ABF shows, as expected, very low contrast. As for the $\mathrm{WS}_{2}$, the iCOM and SSB signals both show good contrast. The distortions visible on the left of the images are due to the slow response of the scan coils at this dwell time as we have no flyback time.

To check if we were able to record 4D STEM scans at dwell times of $100 \mathrm{~ns}$ and that the detector is still able to record a proper 4D STEM dataset, a low magnification scan on a holey carbon film was performed. This sample and magnification was selected to have a high contrast and to minimize the effect of drift since the individual $100 \mathrm{~ns}$ scans have such a low signal-to-noise that aligning them would be very challenging and beyond the scope of the present study. First a $1024 \times 1024$ scan at a $20 \mu$ s dwell time was performed, the bright field (BF) and dark field (DF) images are shown in Fig. 5 b,f). The virtual detectors used to reconstruct the signal are shown in Fig. 5(a,e) where the BF signal arises from the integration over the entire central beam and the DF collects all the signal which lands outside the central beam. Further the same field of view is scanned with the same number of probe positions except the dwell time is decreased to $100 \mathrm{~ns}$ where a total of 200 frames were scanned to have an equal dose as the slower $20 \mu \mathrm{s}$ scan. The resulting summed BF and DF are shown in Fig. 5. (c,g). Large distortions due 
to the finite response of the scan coils are visible but in essence, the 4D STEM signal is recorded properly showing that the detector can handle such short dwell times with ease. In Fig. 5 $(d, h)$ the BF and DF of a single scan are shown where the low signal-to-noise results from the very small number of counts per probe position. These results show that dwell times in the order of $100 \mathrm{~ns}$ would be possible if the scanning system bandwidth could be improved, possibly at the expense of maximum field of view capabilities. Efforts in this direction are reported for magnetic deflection [51] but also electrostatic deflection coupled to high bandwidth amplifiers are a possibility. Beside the practical limitations of the scan system, it is unlikely that we can reduce the dwell time towards the ultimate timing resolution from the Timepix 3 being $1.56 \mathrm{~ns}$ as synchrotron experiments have shown that significant timing inaccuracies can arise increasing this up to $\sim 20 \mathrm{~ns}$ [52]. Nevertheless for any practical TEM setup, the scan engine will currently be the limiting factor by far.

Due to the speed at which full 4D STEM data sets can now be recorded with event based detectors, this technique has the capability to be combined with other conventional high resolution STEM (HR-STEM) methods. For instance, tomographic series can be performed where instead of just the HAADF signal, the full diffraction pattern can be recorded where a large range of scattering angles is available. In the post-processing steps, different signals can be reconstructed to increase the information gathered from the experiment [53] with the potential for even online processing of the datastream [54].

Another method that becomes more attractive for 4D STEM is depth sectioning where instead of the simple fixed focus multi-frame acquisition used in this work, between each frame the focus is changed in order to get three dimensional information about sample [55, 56, 57, 58, 59]. Adding the 4D STEM dataset to such optical sectioning could potentially improve its performance where for instance for S-matrix reconstruction this depth sectioning is necessary [60]. Finally, acquiring 4D STEM data when the scan sequence is changed is easily accessible if one knows at each time where the probe is positioned. These different scanning strategies are used e.g. to decrease damage [61, 62], or distortions [63, 64].

Although the main limit of the current Timepix3 camera is its limited detectable count rate, new plans for a Timepix 4 chip have been revealed where the number of pixels almost quadruples to $512 \times 448$ and the maximum detectable count rate increases by a factor of six. This allows for a current increase by approximately a fac- tor of 24 , bringing it, in the coming years, much more in line with conventional beam currents used in STEM imaging [65].

\section{Conclusion}

In this work, it is shown that using a hybrid pixel direct electron event based detector rather than conventional frame-based cameras enables the recording of 4D STEM datasets with dwell times as low as $100 \mathrm{~ns}$. The detector was characterised at two different acceleration voltages, 60 and $200 \mathrm{kV}$. For $200 \mathrm{kV}$, the maximum electron count rate is half that of $60 \mathrm{kV}$. However when increasing the threshold for $60 \mathrm{kV}$, a significant decrease in collection efficiency is observed. Hence when performing experiments, a compromise on the threshold is required where the higher the threshold, the higher the electron dose rate that can be detected. However this decreases the collection efficiency which is detrimental for beam sensitive materials. Furthermore by synchronizing the detector with a versatile scan engine, multiframe 4D STEM acquisitions could be performed with scan sizes of $1024 \times 1024$ or larger using dwell times in the order of $\mu \mathrm{s}$. This opens up the possibility to always perform 4D STEM acquisition instead of conventional STEM and profit from the significantly higher information content about the sample for the same incoming beam current. In the future, we anticipate improvements in both the data processing times and the maximum count rates detectable by event based detectors to make this type of setup more straightforward.

\section{Acknowledgements}

This project has received funding from the European Union's Horizon 2020 Research Infrastructure Integrating Activities for Advanced Communities under grant agreement No 823717 - ESTEEM3. J.V. and A.B. acknowledge funding from FWO project G093417N ('Compressed sensing enabling low dose imaging in transmission electron microscopy'). J.V. and D.J. acknowledge funding from FWO project G042920N 'Coincident event detection for advanced spectroscopy in transmission electron microscopy'. We acknowledge funding under the European Union's Horizon 2020 research and innovation programme (J.V. and D.J under grant agreement No 101017720, FET-Proactive EBEAM, and C.H., C.G., X.X. and T.J.P. from the European Research Council (ERC) Grant agreement No. 802123-HDEM). 
All data discussed in this manuscript is openly available through Zenodo to stimulate further research on this topic and to improve reproducibility.

\section{References}

[1] S. Procz, C. Avila, J. Fey, G. Roque, M. Schuetz, E. Hamann, X-ray and gamma imaging with medipix and timepix detectors in medical research 127106104. doi:10.1016/j.radmeas.2019.04.007

URL https://linkinghub.elsevier.com/retrieve/ pii/S1350448719300599

[2] B. Bergmann, P. Burian, P. Manek, S. Pospisil, 3d reconstruction of particle tracks in a $2 \mathrm{~mm}$ thick CdTe hybrid pixel detector 79 (2) 165. doi:10.1140/epjc/s10052-019-6673-z URL http://link.springer.com/10.1140/epjc/ s10052-019-6673-z

[3] W. Kuhlbrandt, The resolution revolution 343 (6178) 14431444. doi:10.1126/science.1251652

URL https://www.sciencemag.org/lookup/doi/10. 1126/science.1251652

[4] B. E. Bammes, R. H. Rochat, J. Jakana, D.-H. Chen, W. Chiu, Direct electron detection yields cryo-EM reconstructions at resolutions beyond 3/4 nyquist frequency 177 (3) 589-601. doi:10.1016/j.jsb.2012.01.008

URL https://linkinghub.elsevier.com/retrieve/ pii/S1047847712000287

[5] C. Ophus, Four-dimensional scanning transmission electron microscopy (4d-STEM): From scanning nanodiffraction to ptychography and beyond 25 (3) 563-582. doi:10.1017/S1431927619000497

URL https://www.cambridge.org/core/product/ identifier/S1431927619000497/type/journal_ article

[6] T. J. Pennycook, A. R. Lupini, H. Yang, M. F. Murfitt, L. Jones, P. D. Nellist, Efficient phase contrast imaging in STEM using a pixelated detector. part 1: Experimental demonstration at atomic resolution 151 160-167. doi:10.1016/j.ultramic.2014.09.013

URL https://linkinghub.elsevier.com/retrieve/ pii/S0304399114001934

[7] J. A. Hachtel, J. C. Idrobo, M. Chi, Sub-Ångstrom electric field measurements on a universal detector in a scanning transmission electron microscope 4 (1) 10. doi:10.1186/s40679-018-0059-4

URL https://ascimaging.springeropen.com/ articles/10.1186/s40679-018-0059-4

[8] I. Lazić, E. G. Bosch, S. Lazar, Phase contrast STEM for thin samples: Integrated differential phase contrast 160 265-280. doi:10.1016/j.ultramic.2015.10.011

URL https://linkinghub.elsevier.com/retrieve/ pii/S0304399115300449

[9] H. Yang, T. J. Pennycook, P. D. Nellist, Efficient phase contrast imaging in STEM using a pixelated detector. part II: Optimisation of imaging conditions 151 232-239. doi:10.1016/j.ultramic.2014.10.013

URL https://linkinghub.elsevier.com/retrieve/ pii/S0304399114002058

[10] T. J. Pennycook, G. T. Martinez, P. D. Nellist, J. C. Meyer, High dose efficiency atomic resolution imag\begin{tabular}{ll|lll|} 
ing via electron ptychography & 196 & $131-135$. & doi:
\end{tabular} 10.1016/j.ultramic.2018.10.005

URL https://linkinghub.elsevier.com/retrieve/ pii/S0304399118302316
[11] K. Müller-Caspary, M. Duchamp, M. Rösner, V. Migunov, F. Winkler, H. Yang, M. Huth, R. Ritz, M. Simson, S. Ihle, H. Soltau, T. Wehling, R. E. Dunin-Borkowski, S. Van Aert, A. Rosenauer, Atomic-scale quantification of charge densities in two-dimensional materials 98 (12) 121408. doi:10.1103/PhysRevB.98.121408

URL https://link.aps.org/doi/10.1103/PhysRevB. 98.121408

[12] G. T. Martinez, T. C. Naginey, L. Jones, C. M. O’Leary, T. J. Pennycook, R. J. Nicholls, J. R. Yates, P. D. Nellist, Direct imaging of charge redistribution due to bonding at atomic resolution via electron ptychography arXiv: 1907.12974 URL http://arxiv.org/abs/1907.12974

[13] J. Madsen, T. J. Pennycook, T. Susi, ab initio description of bonding for transmission electron microscopy 11325 doi:10.1016/j.ultramic.2021.113253 URL https://linkinghub.elsevier.com/retrieve/ pii/S0304399121000449

[14] K. Müller-Caspary, F. F. Krause, F. Winkler, A. Béché, J. Verbeeck, S. Van Aert, A. Rosenauer, Comparison of first moment STEM with conventional differential phase contrast and the dependence on electron dose 203 95-104. doi:10.1016/j.ultramic.2018.12.018 URL https://linkinghub.elsevier.com/retrieve/ pii/S0304399118302730

[15] J. Mir, R. Clough, R. MacInnes, C. Gough, R. Plackett, I. Shipsey, H. Sawada, I. MacLaren, R. Ballabriga, D. Maneuski, V. O’Shea, D. McGrouther, A. Kirkland, Characterisation of the medipix3 detector for 60 and $80 \mathrm{keV}$ electrons 182 44-53. doi:10.1016/j.ultramic.2017.06.010

URL https://linkinghub.elsevier.com/retrieve/ pii/S0304399116303989

[16] H. Ryll, M. Simson, R. Hartmann, P. Holl, M. Huth, S. Ihle, Y. Kondo, P. Kotula, A. Liebel, K. MüllerCaspary, A. Rosenauer, R. Sagawa, J. Schmidt, H. Soltau, L. Strüder, A pnCCD-based, fast direct single electron imaging camera for TEM and STEM 11 (4) P04006-P04006. doi:10.1088/1748-0221/11/04/P04006

URL http://stacks.iop.org/1748-0221/ $11 / i=04 / a=P 04006$ ?key=crossref . 341dff1a30df71a36812e65192e3f0f7

[17] C. M. O'Leary, C. S. Allen, C. Huang, J. S. Kim, E. Liberti, P. D. Nellist, A. I. Kirkland, Phase reconstruction using fast binary 4d STEM data 116 (12) 124101. doi:10.1063/1.5143213 URL http://aip.scitation.org/doi/10.1063/1. 5143213

[18] M. Nord, R. W. H. Webster, K. A. Paton, S. McVitie, D. McGrouther, I. MacLaren, G. W. Paterson, Fast pixelated detectors in scanning transmission electron microscopy. part i: Data acquisition, live processing, and storage, Microscopy and Microanalysis 26 (4) (2020) 653-666. doi:10.1017/ S1431927620001713

[19] A. Mayoral, M. Sanchez-Sanchez, A. Alfayate, J. PerezPariente, I. Diaz, Atomic observations of microporous materials highly unstable under the electron beam: The cases of ti-doped alpo4-5 and zn-mof-74 ChemCatChem 7 (22) (2015) 3719-3724. arXiv:https: //chemistry-europe.onlinelibrary.wiley.com/ doi/pdf/10.1002/cctc.201500617 doi:https: //doi.org/10.1002/cctc.201500617 URL https://chemistry-europe.onlinelibrary. wiley.com/doi/abs/10.1002/cctc.201500617

[20] Y. Zhu, J. Ciston, B. Zheng, X. Miao, C. Czarnik, Y. Pan, R. Sougrat, Z. Lai, C.-E. Hsiung, K. Yao, I. Pinnau, M. Pan, Y. Han, Unravelling surface and interfacial structures of a 
metal-organic framework by transmission electron microscopy 16 (5) 532-536. doi:10.1038/nmat4852

URL https://doi.org/10.1038/nmat4852

[21] J. G. Lozano, G. T. Martinez, L. Jin, P. D. Nellist, P. G. Bruce, Low-dose aberration-free imaging of li-rich cathode materials at various states of charge using electron ptychography Nano Letters 18 (11) (2018) 6850-6855, pMID: 30257093. arXiv: https://doi.org/10.1021/acs.nanolett.8b02718 doi:10.1021/acs.nanolett.8b02718

URL https://doi.org/10.1021/acs.nanolett. 8b02718

[22] J. Ciston, I. J. Johnson, B. R. Draney, P. Ercius, E. Fong, A. Goldschmidt, J. M. Joseph, J. R. Lee, A. Mueller, C. Ophus, A. Selvarajan, D. E. Skinner, T. Stezelberger, C. S. Tindall, A. M. Minor, P. Denes, The 4d camera: Very high speed electron counting for 4d-STEM 25 1930-1931. doi:10.1017/S1431927619010389

URL https://www.cambridge.org/core/product/ identifier/S1431927619010389/type/journal_ article

[23] P. Ercius, I. Johnson, H. Brown, P. Pelz, S.-L. Hsu, B. Draney, E. Fong, A. Goldschmidt, J. Joseph, J. Lee, J. Ciston, C. Ophus, M. Scott, A. Selvarajan, D. Paul, D. Skinner, M. Hanwell, C. Harris, P. Avery, T. Stezelberger, C. Tindall, R. Ramesh, A. Minor, P. Denes, The $4 \mathrm{~d}$ camera - an $87 \mathrm{kHz}$ frame-rate detector for counted 4d-STEM experiments 26 1896-1897. doi:10.1017/S1431927620019753

URL https://www. cambridge.org/core/product/ identifier/S1431927620019753/type/journal_ article

[24] J. Song, C. S. Allen, S. Gao, C. Huang, H. Sawada, X. Pan, J. Warner, P. Wang, A. I. Kirkland, Atomic resolution defocused electron ptychography at low dose with a fast, direct electron detector 9 (1) 3919. doi:10.1038/s41598-019-40413-z URL s41598-019-40413-z http://www nature.com/articles/

[25] Z. Chen, M. Odstrcil, Y. Jiang, Y. Han, M.-H. Chiu, L.-J. Li, D. A. Muller, Mixed-state electron ptychography enables sub-angstrom resolution imaging with picometer precision at low dose 11 (1) 2994. doi : 10.1038/s41467-020-16688-6 URL http://www.nature.com/articles/ s41467-020-16688-6

[26] L. Zhou, J. Song, J. S. Kim, X. Pei, C. Huang, M. Boyce, L. Mendonça, D. Clare, A. Siebert, C. S. Allen, E. Liberti, D. Stuart, X. Pan, P. D. Nellist, P. Zhang, A. I. Kirkland, P. Wang, Low-dose phase retrieval of biological specimens using cryo-electron ptychography 11 (1) 2773. doi : 10.1038/s41467-020-16391-6 URL http://www.nature.com/articles/ s41467-020-16391-6

[27] J. Rodenburg, B. McCallum, P. Nellist, Experimental tests on double-resolution coherent imaging via STEM 48 (3) 304-314. doi:10.1016/0304-3991(93)90105-7

URL https://linkinghub.elsevier.com/retrieve/ pii/0304399193901057

[28] The theory of super-resolution electron microscopy via wigner-distribution deconvolution 339 (1655) 521-553. doi:10.1098/rsta.1992.0050

URL https://royalsocietypublishing.org/doi/10. 1098/rsta.1992.0050

[29] H. Yang, R. N. Rutte, L. Jones, M. Simson, R. Sagawa, H. Ryll, M. Huth, T. J. Pennycook, M. Green, H. Soltau, Y. Kondo, B. G. Davis, P. D. Nellist, Simultaneous atomic-resolution electron ptychography and z-contrast imaging of light and heavy elements in complex nanostructures 7 (1) 12532. doi:10.1038/ ncomms12532

URL http://www.nature.com/articles/ncomms12532

[30] P. Hatfield, W. Furnell, A. Shenoy, E. Fox, R. Parker, L. Thomas, The LUCID-timepix spacecraft payload and the CERN@school educational programme 13 (10) C10004-C10004. doi:10. 1088/1748-0221/13/10/C10004

URL https://iopscience.iop.org/article/10.1088/ 1748-0221/13/10/C10004

[31] R. Beacham, A. M. Raighne, D. Maneuski, V. O'Shea, S. McVitie, D. McGrouther, Medipix2/timepix detector for time resolved transmission electron microscopy 6 (12) C12052C12052. doi:10.1088/1748-0221/6/12/C12052 URL https://iopscience.iop.org/article/10.1088/ 1748-0221/6/12/C12052

[32] Advacam advapix TPX3 URL https://advacam.com/camera/advapix-tpx3

[33] F. F. Krause, M. Schowalter, O. Oppermann, D. Marquardt, K. Müller-Caspary, R. Ritz, M. Simson, H. Ryll, M. Huth, H. Soltau, A. Rosenauer, Precise measurement of the electron beam current in a TEM 223113221. doi:10.1016/j.ultramic.2021.113221

URL https://linkinghub.elsevier.com/retrieve/ pii/S0304399121000164

[34] A. Zobelli, S. Y. Woo, A. Tararan, L. H. Tizei, N. Brun, X. Li, O. Stéphan, M. Kociak, M. Tencé, Spatial and spectral dynamics in STEM hyperspectral imaging using random scan patterns 112912doi : 10.1016/j.ultramic. 2019.112912 URL https://linkinghub.elsevier.com/retrieve/ pii/S0304399119303110

[35] Attolight URL https : //attolight.com/

[36] D. Jannis, C. Hofer, C. Gao, X. Xie, A. Beche, T. Pennycook, J. Verbeeck, Event driven 4d STEM acquisition with a timepix 3 detector: microsecond dwelltime and faster scans for high precision and low dose applicationsPublisher: Zenodo. doi: $10.5281 /$ zenodo.5068510

[37] A. Rosenfeld, M. Silari, M. Campbell, The editorial 139 106483. doi:10.1016/j.radmeas.2020.106483 URL https://linkinghub.elsevier.com/retrieve/ pii/S1350448720302596

[38] R. Ballabriga, M. Campbell, X. Llopart, Asic developments for radiation imaging applications: The medipix and timepix family 878 10-23. doi:10.1016/j.nima.2017.07.029

URL https://linkinghub.elsevier.com/retrieve/ pii/S0168900217307714

[39] J. P. van Schayck, E. van Genderen, E. Maddox, L. Roussel, H. Boulanger, E. Fröjdh, J.-P. Abrahams, P. J. Peters, R. B. Ravelli, Sub-pixel electron detection using a convolutional neural network 218113091. doi:10.1016/j.ultramic.2020.113091

URL https://linkinghub.elsevier.com/retrieve/ pii/S0304399120302424

[40] J. Jakubek, Precise energy calibration of pixel detector working in time-over-threshold mode Nuclear Instruments and Methods in Physics Research Section A: Accelerators, Spectrometers, Detectors and Associated Equipment 633 (2011) S262-S266, 11th International Workshop on Radiation Imaging Detectors (IWORID). doi:https://doi.org/10.1016/j.nima.2010.06.183 URL https://www.sciencedirect.com/science/ article/pii/S0168900210013732

[41] M. Kroupa, T. Campbell-Ricketts, A. Bahadori, A. Empl, Techniques for precise energy calibration of particle pixel detectors Review of Scientific Instruments 88 (3) (2017) 033301. arXiv : https://doi.org/10.1063/1.4978281 doi:10.1063/1. 
4978281

URL https://doi.org/10.1063/1.4978281

[42] M. Urban, D. Doubravová, Timepix3: Temperature influence on X-ray measurements in counting mode with si sensor Radiation Measurements 141 (2021) 106535. doi:https: //doi.org/10.1016/j.radmeas.2021.106535

URL https://www.sciencedirect.com/science/ article/pii/S1350448721000184

[43] D. Jannis, K. Müller-Caspary, A. Béché, J. Verbeeck, Coincidence detection of eels and edx spectral events in the electron microscope Applied Sciences 11 (19) (2021). doi:10.3390/ app11199058

URL https : //www . mdpi.com/2076-3417/11/19/9058

[44] K. A. Paton, M. C. Veale, X. Mu, C. S. Allen, D. Maneuski, C. Kübel, V. O'Shea, A. I. Kirkland, D. McGrouther, Quantifying the performance of a hybrid pixel detector with gaas:cr sensor for transmission electron microscopy Ultramicroscopy 227 (2021) 113298. doi:https: //doi.org/10.1016/j.ultramic.2021.113298

URL https://www.sciencedirect.com/science/ article/pii/S0304399121000851

[45] H. Guo, E. Franken, Y. Deng, S. Benlekbir, G. Singla Lezcano, B. Janssen, L. Yu, Z. A. Ripstein, Y. Z. Tan, J. L. Rubinstein, Electron-event representation data enable efficient cryoEM file storage with full preservation of spatial and temporal resolution 7 (5) 860-869. doi : 10.1107/S205225252000929X URL https://scripts.iucr.org/cgi-bin/paper? S205225252000929X

[46] A. Datta, K. F. Ng, D. Balakrishnan, M. Ding, S. W. Chee, Y. Ban, J. Shi, N. D. Loh, A data reduction and compression description for high throughput time-resolved electron microscopy 12 (1) 664. doi : 10.1038/s41467-020-20694-z URL http://www.nature.com/articles/ s41467-020-20694-z

[47] P. M. Pelz, I. Johnson, C. Ophus, P. Ercius, M. C. Scott, Realtime interactive 4d-STEM phase-contrast imaging from electron event representation datar $\operatorname{ar}$ iv : 2104.06336 URL http://arxiv.org/abs/2104.06336

[48] B. H. Savitzky, I. El Baggari, C. B. Clement, E. Waite, B. H. Goodge, D. J. Baek, J. P. Sheckelton, C. Pasco, H. Nair, N. J. Schreiber, J. Hoffman, A. S. Admasu, J. Kim, S.-W. Cheong, A. Bhattacharya, D. G. Schlom, T. M. McQueen, R. Hovden, L. F. Kourkoutis, Image registration of low signal-to-noise cryo-STEM data 191 56-65. doi:10.1016/j.ultramic.2018.04.008

URL https://linkinghub.elsevier.com/retrieve/ pii/S0304399117304369

[49] C. M. O’Leary, G. T. Martinez, E. Liberti, M. J. Humphry, A. I Kirkland, P. D. Nellist, Contrast transfer and noise considerations in focused-probe electron ptychography 221113189. doi:10.1016/j.ultramic.2020.113189

URL https://linkinghub.elsevier.com/retrieve/ pii/S0304399120303314

[50] S. Fujiyama, K. Yoza, N. Kamiya, K. Nishi, Y. Yokomori, Entrance and diffusion pathway of $\mathrm{CO}_{2}$ and dimethyl ether in silicalite-1 zeolite channels as determined by single-crystal XRD structural analysis 71 (1) 112-118. doi:10.1107/S2052520615000256

URL http://scripts.iucr.org/cgi-bin/paper? S2052520615000256

[51] R. Ishikawa, Y. Jimbo, M. Terao, M. Nishikawa, Y. Ueno, S. Morishita, M. Mukai, N. Shibata, Y. Ikuhara, High spatiotemporal-resolution imaging in the scanning transmission electron microscope 69 (4) 240-247. doi:10.1093/jmicro/ dfaa017
URL https://academic.oup.com/jmicro/article/69/ 4/240/5815181

[52] G. Crevatin, D. Omar, I. Horswell, H. Yousef, E. N. Gimenez, N. Tartoni, Timepix3 readout system for time resolved experiments at synchrotron radiation facilities, in: 2016 IEEE Nuclear Science Symposium, Medical Imaging Conference and Room-Temperature Semiconductor Detector Workshop (NSS/MIC/RTSD), 2016, pp. 1-5. doi:10.1109/NSSMIC. 2016.8069823

[53] D. J. Chang, D. S. Kim, A. Rana, X. Tian, J. Zhou, P. Ercius, J. Miao, Ptychographic atomic electron tomography: Towards three-dimensional imaging of individual light atoms in materials 102 (17) 174101. doi:10.1103/PhysRevB.102.174101 URL https://link.aps.org/doi/10.1103/PhysRevB. 102.174101

[54] A. Clausen, D. Weber, K. Ruzaeva, V. Migunov, A. Baburajan, A. Bahuleyan, J. Caron, R. Chandra, S. Dey, S. Halder, D. S. Katz, B. D. Levin, M. Nord, C. Ophus, S. Peter, J. Schyndel van, J. Shin, S. Sunku, K. Müller-Caspary, R. E. Dunin-Borkowski, LiberTEM/LiberTEM: 0.7.0 doi:10. 5281/ZENOD0.4923277 URL https : //zenodo.org/record/4923277

[55] K. van Benthem, A. R. Lupini, M. Kim, H. S. Baik, S. Doh, J.-H. Lee, M. P. Oxley, S. D. Findlay, L. J. Allen, J. T. Luck, S. J. Pennycook, Three-dimensional imaging of individual hafnium atoms inside a semiconductor device 87 (3) 034104. doi:10.1063/1.1991989

URL http://aip.scitation.org/doi/10.1063/1. 1991989

[56] R. Ishikawa, S. J. Pennycook, A. R. Lupini, S. D. Findlay, N. Shibata, Y. Ikuhara, Single atom visibility in STEM optical depth sectioning 109 (16) 163102. doi:10.1063/1.4965709 URL http://aip.scitation.org/doi/10.1063/1. 4965709

[57] H. L. Xin, D. A. Muller, Aberration-corrected ADF-STEM depth sectioning and prospects for reliable $3 \mathrm{~d}$ imaging in $\mathrm{s} / \mathrm{TEM}$ 58 (3) 157-165. doi:10.1093/jmicro/dfn029

URL https://academic.oup.com/jmicro/ article-lookup/doi/10.1093/jmicro/dfn029

[58] T. J. Pennycook, H. Yang, L. Jones, M. Cabero, A. RiveraCalzada, C. Leon, M. Varela, J. Santamaria, P. D. Nellist, 3d elemental mapping with nanometer scale depth resolution via electron optical sectioning 174 27-34. doi:10.1016/j.ultramic.2016.12.002

URL https://linkinghub.elsevier.com/retrieve/ pii/S0304399116303631

[59] H. Yang, J. G. Lozano, T. J. Pennycook, L. Jones, P. B. Hirsch, P. D. Nellist, Imaging screw dislocations at atomic resolution by aberration-corrected electron optical sectioning 6 (1) 7266. doi:10.1038/ncomms8266 URL http://www .nature.com/articles/ncomms8266

[60] P. M. Pelz, H. G. Brown, J. Ciston, S. D. Findlay, Y. Zhang, M. Scott, C. Ophus, Reconstructing the scattering matrix from scanning electron diffraction measurements alone $\operatorname{arXiv}: 2008$. 12768

URL http://arxiv.org/abs/2008.12768

[61] A. Velazco, D. Jannis, A. Béché, J. Verbeeck, Reducing electron beam damage through alternative STEM scanning strategies. part i - experimental findings arXiv: 2105.01617 URL http://arxiv.org/abs/2105.01617

[62] D. Nicholls, J. Lee, H. Amari, A. J. Stevens, B. L. Mehdi, N. D. Browning, Minimising damage in high resolution scanning transmission electron microscope images of nanoscale structures and processes 12 (41) 21248-21254. doi : 10.1039/ DONR04589F 
URL http://xlink.rsc.org/?DOI=DONR04589F

[63] X. Sang, A. R. Lupini, R. R. Unocic, M. Chi, A. Y. Borisevich, S. V. Kalinin, E. Endeve, R. K. Archibald, S. Jesse, Dynamic scan control in STEM: spiral scans 2 (1) 6. doi:10.1186/s40679-016-0020-3

URL https://ascimaging.springeropen.com/ articles/10.1186/s40679-016-0020-3

[64] A. Velazco, M. Nord, A. Béché, J. Verbeeck, Evaluation of different rectangular scan strategies for STEM imaging 215 113021. doi:10.1016/j.ultramic.2020.113021

URL https://linkinghub.elsevier.com/retrieve/ pii/S0304399120300334

[65] Xavier Llopart, Timepix4 detectors

URL https://indico.cern.ch/event/876275/ contributions/3729426/attachments/1986038/

3309319/Xavi_Timepix4_Muon.pdf 


\section{Supplementary Material}

\section{S1. Energy threshold variation for flat field illumination}

In Fig 6, the average cluster size as a function of threshold is shown. At low thresholds from 1-5 keV the cluster size decreases with decreasing threshold. This might seem nonphysical since the lower the threshold the larger the cluster size induced by an electron hit should be. However, one possible explanation for this effect would be that at low thresholds $\leq 5 \mathrm{keV}$ thermal noise or other types of random noise start to be detected resulting in a smaller average cluster size since the average cluster size of random noise is one, although we note that at $1 \mathrm{kV}$ the grid pattern artefact that appears in the flat field images is likely due to the underlying detector geometry. The results of flat field illumination of the incoming electron beam at 60 and $200 \mathrm{kV}$ accelerating voltages are shown in Fig. 7 and 8 as a function of the threshold voltage. In order to remove the influence of such noise, the minimum usable threshold was determined to be at $7 \mathrm{keV}$. From Fig. 7 and 8 , a maximum value of the threshold is determined by investigating the number of dead pixels as a function of threshold. A dead pixel is defined as a pixel which is not giving any counts during the acquisition. At $35 \mathrm{keV}$ a sharp increase in the number of dead pixels is seen making higher threshold values undesirable for the experiments. Therefore the valid range of thresholds is determine to be between 7 and $33 \mathrm{keV}$.

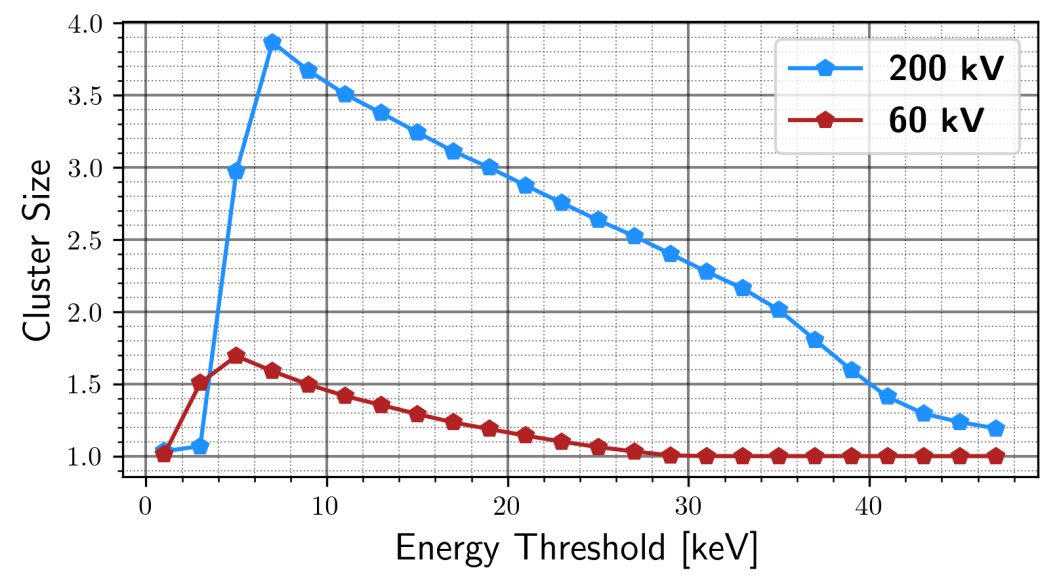

Figure 6: The average cluster size calculated as a function of threshold for 60 and $200 \mathrm{kV}$ accelerating voltages.

\section{S2. Raw vs declustered 4D STEM dataset}

In this section, we investigate if the clustering of the electron events has a significant influence on the reconstructed integrated centre-of-mass (iCOM) signal. An electron probe was scanned with a $6 \mu$ s dwell time over $1024 \times 1024$ points of a zeolite silicalite- 1 sample at a $200 \mathrm{kV}$ accelerating voltage. The declustering algorithm searches for adjacent pixels which are excited within a time interval of $100 \mathrm{~ns}$ [39]. From this cluster the new corrected time-ofarrival (TOA) is when the first pixel of the cluster is excited. The point of impact is calculated using the centre-of-mass of the cluster. In Fig. $9(\mathrm{a}, \mathrm{b})$, respectively the raw and declustered reconstructed iCOM images are shown and seen to be very similar. The difference between the two signals is shown in Fig. 9 (c), showing that for iCOM the reduction of modulation transfer function (MTF) has no visual influence on the result. The average value of the difference is $1 \%$. Hence when the $\mathrm{iCOM}$ signal is used to extract atomic positions, declustering will not greatly improve the data quality. However, when quantitative information is desired from the iCOM signal, declustering is expected to improve the results. In Fig. 9(d), 500 events collected sequentially from the dataset are shown from which the clusters are clearly visible. In (e), the corrected events are shown with the estimated points of impact of the electrons. In the inset figures the position averaged convergent beam electron diffraction (PACBED) of both raw and declustered datasets are shown in which also no clear difference is observed. 


\section{$60 \mathrm{kV}$}

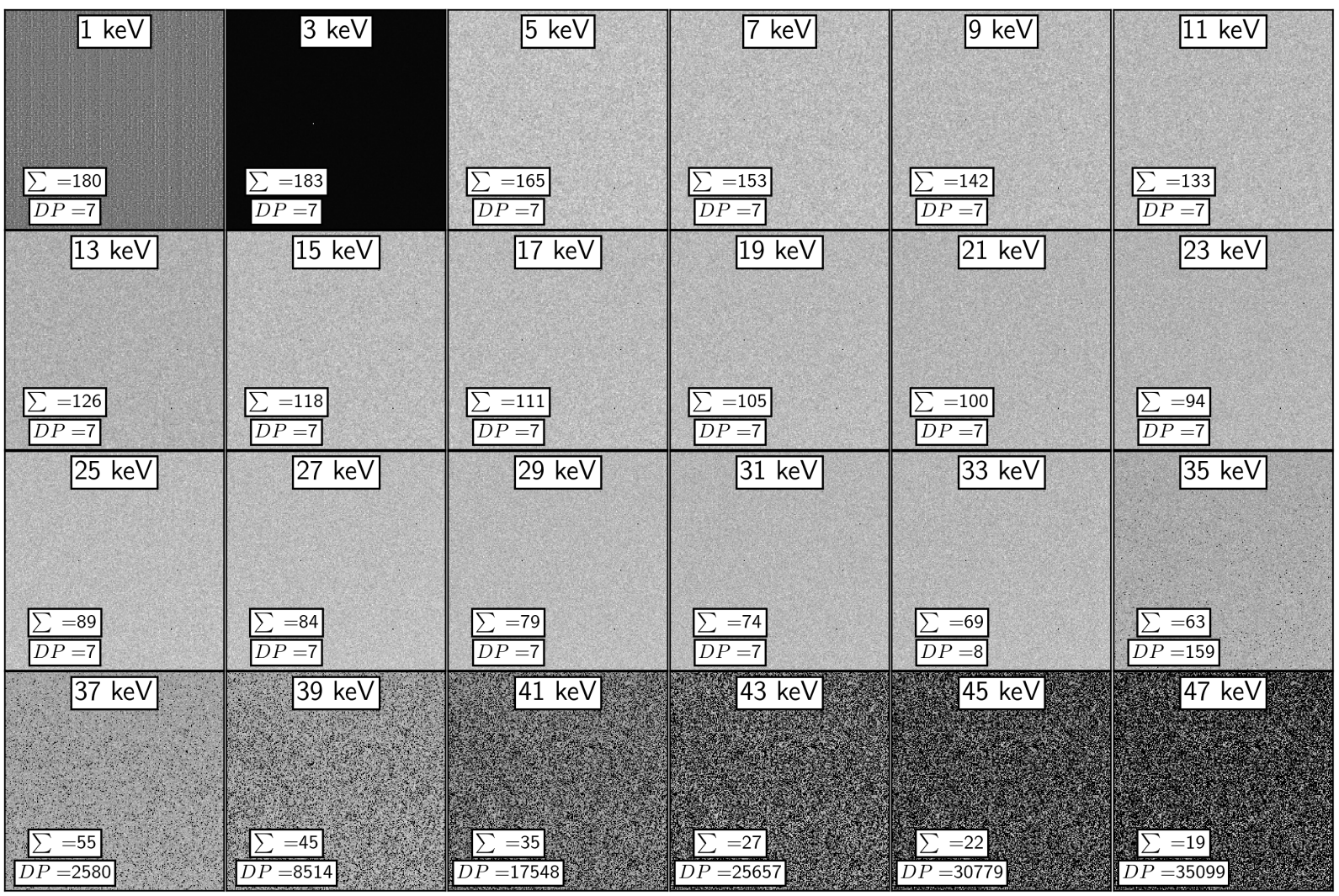

Figure 7: The flat field illumination of $60 \mathrm{kV}$ electrons where the threshold is varied. The relative total number of detected electrons compared to $21 \mathrm{keV}$ in percentage is shown and also the number of dead pixels is indicated in the plots.

\section{S3. Artefacts in Timepix3 data}

When recording a data stream we find that sometimes the camera does not record the incoming electrons for certain time periods ranging from $50 \mu$ s to $1.75 \mathrm{~ms}$. To investigate this, the dataset of the low magnification scan used for Fig. 5 of the main text is used. In Fig. 10 (a), a histogram calculated from the TOA of the incoming events with a bin size of $10 \mu \mathrm{s}$ is shown. This shows the number of events where at some particular times, the counts drop unphysicaly to zero. The time that the camera is down for this acquisition is $0.12 \%$, which is a relatively small proportion of time compared to the entire acquisition. In Fig. 10 (b), the corresponding dark field (DF) image is shown where the virtual detector region is shown in Fig. 5. The black lines on the image are due to the artefacts. In this work a very simple method is used to mitigate the artefacts by filling these pixels with the average value of the image. While this is likely not the best way to reconstruct the images, this is beyond the scope of our present work. In the future we expect other improved methods for image restoration or ideally ways to avoid the artefacts in the first place can be developed. 
$200 \mathrm{kV}$

\begin{tabular}{|c|c|c|c|c|c|}
\hline $1 \mathrm{keV}$ & $3 \mathrm{keV}$ & $5 \mathrm{keV}$ & $7 \mathrm{keV}$ & $9 \mathrm{keV}$ & $11 \mathrm{keV}$ \\
\hline $\begin{array}{l}\sum=148 \\
D P=7\end{array}$ & $\begin{array}{l}\sum=148 \\
D P=7\end{array}$ & $\begin{array}{l}\sum=147 \\
D P=7\end{array}$ & $\begin{array}{l}\sum=137 \\
D P=7\end{array}$ & \begin{tabular}{|l|l|}
$\sum=130$ \\
$D P=7$
\end{tabular} & \begin{tabular}{|l}
$\sum=124$ \\
$D P=7$
\end{tabular} \\
\hline $13 \mathrm{keV}$ & $15 \mathrm{keV}$ & $17 \mathrm{keV}$ & $19 \mathrm{keV}$ & $21 \mathrm{keV}$ & $23 \mathrm{keV}$ \\
\hline \begin{tabular}{|l|}
$\sum=119$ \\
$D P=7$ \\
$D$
\end{tabular} & \begin{tabular}{|l|}
$\sum=113$ \\
$D P=7$ \\
\end{tabular} & $\begin{array}{l}\sum=108 \\
D P=7\end{array}$ & $\begin{array}{l}\sum=104 \\
D P=7\end{array}$ & $\begin{array}{l}\sum=100 \\
D P=7\end{array}$ & $\begin{array}{l}\sum=95 \\
D P=7\end{array}$ \\
\hline $25 \mathrm{keV}$ & $27 \mathrm{keV}$ & $29 \mathrm{keV}$ & $31 \mathrm{keV}$ & $33 \mathrm{keV}$ & $35 \mathrm{keV}$ \\
\hline \begin{tabular}{|l|}
$\sum=91$ \\
$D P=7$
\end{tabular} & $\begin{array}{l}\sum=87 \\
D P=7 \\
D=1\end{array}$ & $\begin{array}{l}\sum=82 \\
D P=7\end{array}$ & $\begin{array}{l}\sum=78 \\
D P=7\end{array}$ & \begin{tabular}{|l|}
$\sum=74$ \\
$D P=7$ \\
\end{tabular} & \begin{tabular}{|l|}
$\sum \sum=68$ \\
$D P=49$
\end{tabular} \\
\hline $37 \mathrm{keV}$ & $39 \mathrm{keV}$ & $41 \mathrm{keV}$ & $43 \mathrm{keV}$ & $45 \mathrm{keV}$ & $47 \mathrm{keV}$ \\
\hline$\frac{\sum=61}{D P=1270}$ & $\frac{\sum=53}{D P=5555}$ & $\frac{\mid \sum=44}{D P=12810}$ & $\begin{array}{l}\sum=36 \\
D P=19809\end{array}$ & $\begin{array}{l}\sum=32 \\
D P=2465\end{array}$ & $\begin{array}{c}\sum=28 \\
D P=29052\end{array}$ \\
\hline
\end{tabular}

Figure 8: The flat field illumination of $200 \mathrm{kV}$ electrons where the threshold is varied. The relative total number of detected electrons compared to $21 \mathrm{keV}$ in percentage is shown and also the number of dead pixels is indicated in the plots.
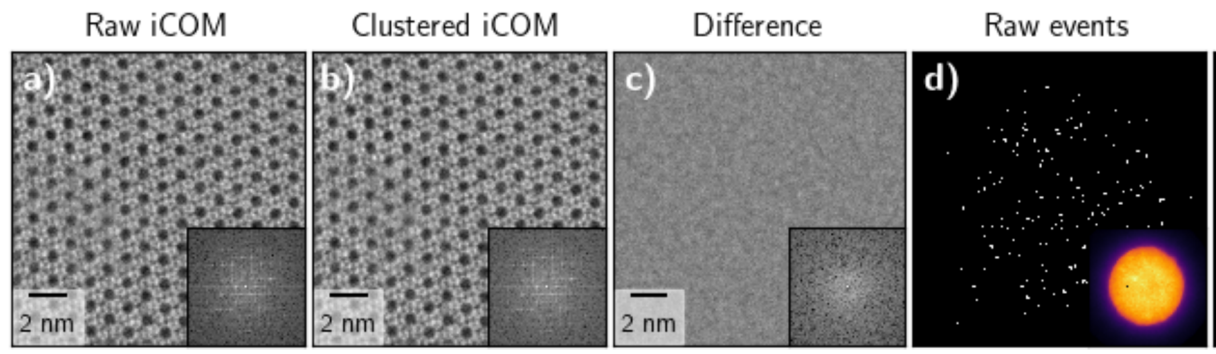

Declustered events

Figure 9: (a) The reconstructed iCOM image of the raw dataset from a silicalite- 1 zeolite. The scan size is $1024 \times 1024$, the dwell time is $6 \mu$ s, and the acceleration voltage is $200 \mathrm{kV}$. In the insets the Fourier transform of the iCOM signal is shown. (b) The same as (a) except declustering is applied. (c) The difference between (a) and (b) showing the similarity between the two signals. (d) a set of 500 sequential events from the same dataset detected on the Timepix 3 camera, in which the declustered event is clearly seen. In the inset the PACBED pattern is shown. (e) The same as (d) but now with declustering applied. 


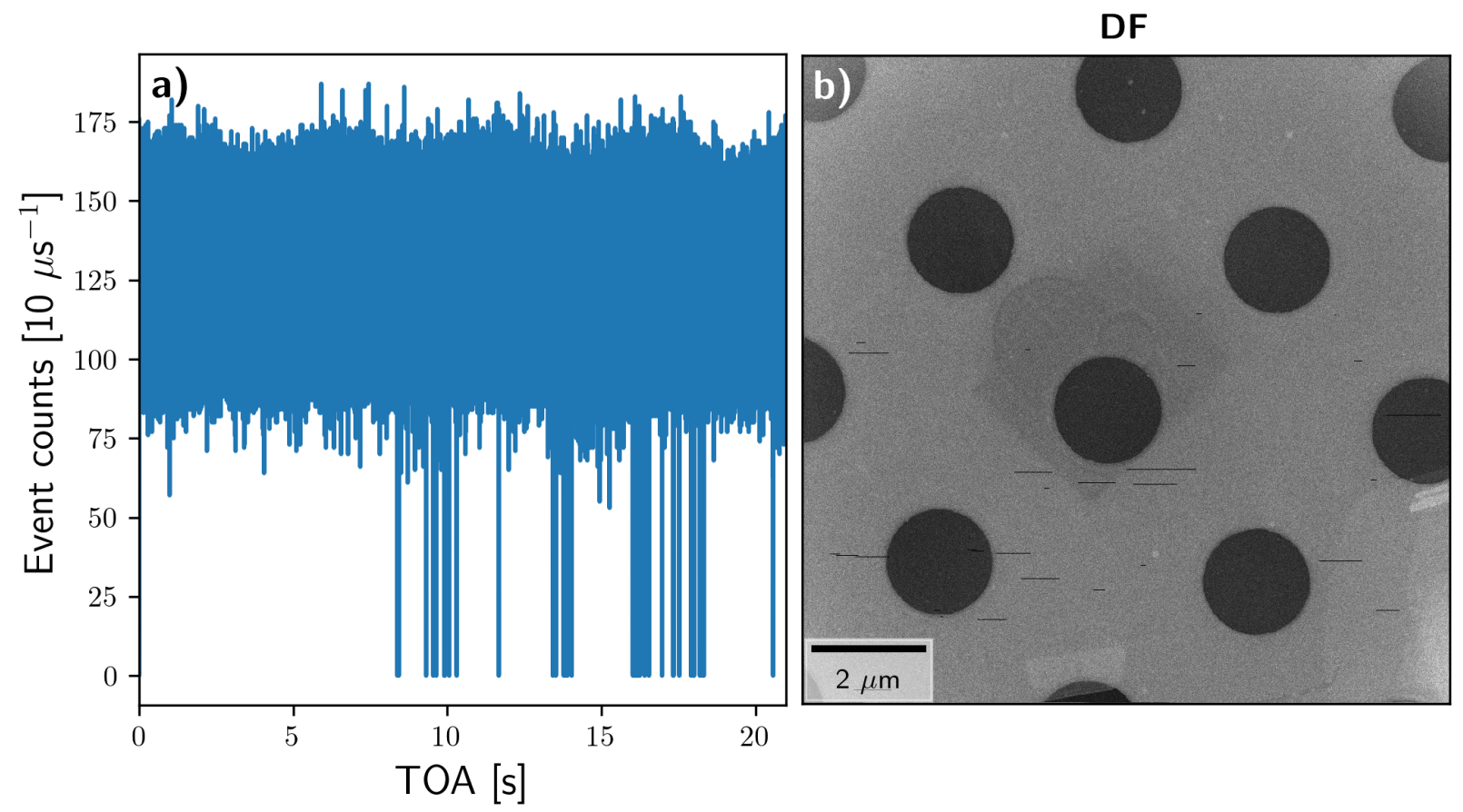

Figure 10: (a) A histogram of the TOA of the incoming events with a bin size of $10 \mu \mathrm{s}$. (b) The reconstructed DF signal where the dark lines correspond to the artefacts which arise when the detector does not detect signal in some time windows. 